\title{
Prevention of Thromboembolic Events in Patients with COVID-19
}

\author{
Surbhi Warrior ${ }^{110}$ Elizabeth Behrens ${ }^{1}$ Joshua Thomas $^{1}$ Sefer Gezer ${ }^{2}$ \\ Parameswaran Venugopal $^{2}$ Shivi Jain ${ }^{2}$
}

${ }^{1}$ Department of Internal Medicine, Rush University Medical Center, Chicago, Illinois, United States

2 Division of Hematology-Oncology, Rush University Cancer Center, Chicago, Illinois, United States

Address for correspondence Surbhi Warrior, MD, MPH, Department of Internal Medicine, Rush University Medical Center, 1620 West Harrison Street, Chicago, IL 60612, United States

(e-mail: surbhi_warrior@rush.edu).

TH Open 2021;5:e415-e419.

The novel coronavirus disease 2019 (COVID-19)-associated coagulopathy is a known cause of significant morbidity and mortality in patients affected by coronavirus. The proposed pathogenesis of hypercoagulability in COVID-19 patients is attributed to Virchow's Triad, wherein hypoxia, severe inflammation, and cytokine storm cause endothelial injury, activation of the coagulation cascade, and immobilization in critically ill patients, all contribute to increased risk of thrombosis. ${ }^{1}$ Patients requiring intensive care unit (ICU) admission have increased risk of thrombosis despite standard dose of thromboprophylaxis. ${ }^{2-4}$ COVID-19 treatment has focused on targeting the unregulated inflammatory state to decrease incidence of COVID-19-related complications, including thrombosis.

Due to increased risk of thromboembolism, prophylactic anticoagulation is recommended in all hospitalized COVID19 patients and intermediate to therapeutic dosing is suggested in patients with severe COVID-19, such as those requiring ICU admission. ${ }^{5,6}$ In addition to hypercoagulability, COVID-19 patients are also at an increased risk for bleeding events due to variance in platelet production and destruction, consumption of coagulation factors in the setting of severe inflammation, increased exposure to and dosing of anticoagulation, and requirement of renal replacement therapy (RRT), indicating the need for further investigation into the long-term effect of escalated thromboprophylaxis dosing in this patient population. ${ }^{7,8}$

This analysis was done to better understand the prevention of thrombosis in hospitalized COVID-19 patients by way of analyzing risk factors, impact of anticoagulation, and how COVID-19-specific therapies played a role in incidence of thrombosis and mortality. Adult patients with COVID-19 between March 1, 2020, and June 26, 2020, hospitalized at

received

February 10, 2021

accepted after revision

July 27, 2021 our institution were included in this retrospective analysis. Thrombotic events, including deep vein thrombosis (DVT), pulmonary embolism (PE), and stroke, were assessed by imaging such as leg compression ultrasounds, computed tomography (CT) pulmonary angiography, and head CT or magnetic resonance imaging (MRI), respectively, for patients who had signs and symptoms of venous thromboembolism (VTE) during hospitalization for COVID-19 to confirm the presence of thromboembolism.

Out of a total of 1,265 COVID-19-positive hospitalized patients, there were 323 patients with signs and symptoms for thromboembolism, defined by getting a CT head or MRI, CT pulmonary angiography, or leg compression ultrasounds. Of all COVID-19-positive hospitalized patients, 138 (10.9\%) patients had a confirmed thromboembolism with characteristics shown in - Table 1. Subgroup analysis on incidence of thromboses in all COVID-19 patients showed no difference between gender (male, $11.6 \%$ and female, $9.9 \% ; p=0.3375$ ), race and ethnicity (African American, 11.8\%; Hispanic, 9.4\%; other, $18.4 \%$; White, $9.4 \% ; p=0.0899$ ), or body mass index (BMI; normal defined as $<25 \mathrm{~kg} / \mathrm{m}^{2}$ at $8.9 \%$, overweight defined as $25-30 \mathrm{~kg} / \mathrm{m}^{2}$ at $11.17 \%$, obese defined as $>30 \mathrm{~kg} / \mathrm{m}^{2}$ at $12.6 \%$; $p=0.2183$ ). The average age of COVID-19 patients with thrombosis was significantly greater than the average age of COVID19 patients without thrombosis, 59.6 years versus 57.2 years $(p=0.0005)$. Hispanic patients with thrombosis had a significantly higher mortality rate of $51 \%$ compared with mortality rate of other groups with thrombosis: African American, 18\%; other, $29 \%$; or White, $20 \%$ ( $p=0.0020$ ).

Incidence of thrombosis in ICU patients with COVID-19 was significantly greater than in non-ICU patients with COVID-19, 23.9 versus $2.95 \%(p<0.0001)$. COVID-19 patients who had thrombosis had significantly higher need for

(C) 2021. The Author(s).

This is an open access article published by Thieme under the terms of the Creative Commons Attribution License, permitting unrestricted use, distribution, and reproduction so long as the original work is properly cited. (https://creativecommons.org/licenses/by/4.0/)

Georg Thieme Verlag KG, Rüdigerstraße 14, 70469 Stuttgart, Germany 
Table 1 Patient characteristics of COVID-19 patients with thrombosis

\begin{tabular}{|c|c|}
\hline Characteristics & $\begin{array}{l}\text { Thrombosis } n=138 \\
n(\%)\end{array}$ \\
\hline $\begin{array}{l}\text { Age }(y) \\
\text { Mean (range) }\end{array}$ & $59.6(23-89)$ \\
\hline Young $<45$ & $18(13.0)$ \\
\hline Middle aged (45-65) & $78(56.5)$ \\
\hline Elderly $>65$ & $42(30.4)$ \\
\hline \multicolumn{2}{|l|}{ Sex } \\
\hline Male & $82(59.4)$ \\
\hline Female & $56(40.5)$ \\
\hline \multicolumn{2}{|l|}{ Race and ethnicity } \\
\hline White & $15(10.9)$ \\
\hline African American & $56(40.5)$ \\
\hline Hispanic & $51(36.8)$ \\
\hline Other & $16(11.6)$ \\
\hline \multicolumn{2}{|l|}{ BMI $\left(\mathrm{kg} / \mathrm{m}^{2}\right)$} \\
\hline Normal $(<24.9)$ & $36(26.1)$ \\
\hline Overweight (25-29.9) & $41(29.7)$ \\
\hline Obese $(>30)$ & $56(40.5)$ \\
\hline Unknown & $5(3.6)$ \\
\hline \multicolumn{2}{|l|}{ Thromboembolism type $^{a}$} \\
\hline Deep vein thrombosis & $71(51.4)$ \\
\hline Pulmonary embolism & $61(44.2)$ \\
\hline Stroke & $26(18.8)$ \\
\hline \multicolumn{2}{|c|}{ Anticoagulation prior to thrombosis } \\
\hline None & $30(21.7)$ \\
\hline Prophylactic & $66(47.8)$ \\
\hline Enoxaparin & $43(31.2)$ \\
\hline Heparin & $23(16.7)$ \\
\hline Therapeutic & $41(29.7)$ \\
\hline Enoxaparin & $29(21.0)$ \\
\hline Heparin & $5(3.6)$ \\
\hline Apixaban & $4(2.9)$ \\
\hline Bivalirudin & $3(2.2)$ \\
\hline Unknown & $1(0.7)$ \\
\hline \multicolumn{2}{|l|}{ COVID-19-related therapyb } \\
\hline Steroids & $64(46.4)$ \\
\hline Tocilizumab & $36(26.1)$ \\
\hline Remdesivir & $18(13.0)$ \\
\hline Hydrocychloroquine & $48(34.8)$ \\
\hline Mortality & 44 (31.9) \\
\hline \multicolumn{2}{|l|}{ Hospital characteristics } \\
\hline $\begin{array}{l}\text { Length of stay } \\
\text { Mean (range) }\end{array}$ & $20.5(1-92)$ \\
\hline ICU admission & $116(84.1)$ \\
\hline
\end{tabular}

Table 1 (Continued)

\begin{tabular}{|l|l|}
\hline Characteristics & $\begin{array}{l}\text { Thrombosis } \boldsymbol{n}=\mathbf{1 3 8} \\
\boldsymbol{n}(\%)\end{array}$ \\
\hline $\begin{array}{l}\text { ICU length of stay } \\
\text { Mean (range) }\end{array}$ & $18.7(0.08-91.2)$ \\
\hline Mechanical ventilation & $98(71)$ \\
\hline D-dimer $>6$ & $68(49.2)$ \\
\hline Renal replacement therapy & $45(32.6)$ \\
\hline
\end{tabular}

Abbreviations: BMI, body mass index; COVID-19, novel coronavirus disease 2019; ICU, intensive care unit;

aSome patients had multiple types of thromboembolism.

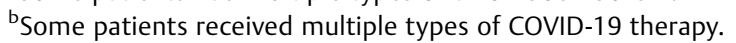

intubation and mechanical ventilation compared with COVID-19 patients who did not have thrombosis, 71.0 versus $24.8 \%(p=0.00001)$. Incidence of thrombosis in COVID-19 patients with D-dimer levels $>6 \mathrm{x}$ ULN was significantly greater than COVID-19 patients who had lower D-dimer levels, 36.6 versus $8.2 \%(p<0.0001)$. The mortality rate of patients with thrombosis who received RRT was significantly higher than those who did not require RRT, 47.4 versus $26.0 \%$ $(p=0.0161)$. Mortality rate in COVID-19 patients with thrombosis was significantly higher than COVID-19 patients without thrombosis, 31.9 versus $10 \%(p<0.0001)$. The average hospital length of stay (LOS) in patients with thrombosis was significantly higher than hospital LOS in patients without thrombosis, 20.6 versus 9.8 days $(p<0.0001)$.

Among the COVID-19-positive patients who had thrombosis, we studied the impact of COVID-19 directed therapy on the incidence of thrombosis. Analysis showed that the incidence of thrombosis in COVID-19 patients who received steroids was significantly less at $14 \%$ as compared with incidence of thrombosis in other COVID-19 treatment: tocilizumab, $25 \% \quad(p=0.0031)$; hydroxychloroquine, $42 \%$ $(p<0.0001)$; and remdesivir, $72 \%(p<0.0001)$. A Cox's regression model showed that when adjusting for age, race, ethnicity, and BMI for demographics and ICU admission for severity of disease, there was no correlation between patients who received steroids compared with those who did not receive steroids to incidence of thrombosis (hazard ratio $[H R]=0.959, p=0.8452$ ).

Adjusting for demographics, as well as ICU admission status to account for severity of disease, a Cox's regression model showed that there was no difference in mortality between COVID-19 patients who received therapeutic versus prophylactic anticoagulation prior to diagnosis of thrombosis $(H R=1.0538, p=0.8831)$. The bleeding rate in COVID-19 patients with thrombosis was significantly higher than reported bleeding rates for hospitalized nonCOVID-19 patients on anticoagulants, 12.3 versus $7.2 \%(p<0.05))^{5,9}$ The majority of bleeding events occurred after a noted thrombosis (58.8\%) on therapeutic dosing of anticoagulation (64.7\%), in the ICU (70.6\%), and were World health Organization (WHO) grade $4(58.8 \%$ ) as seen in - Table 2 . Only $17.6 \%$ of COVID-19 patients with thrombosis who had bleeding had 


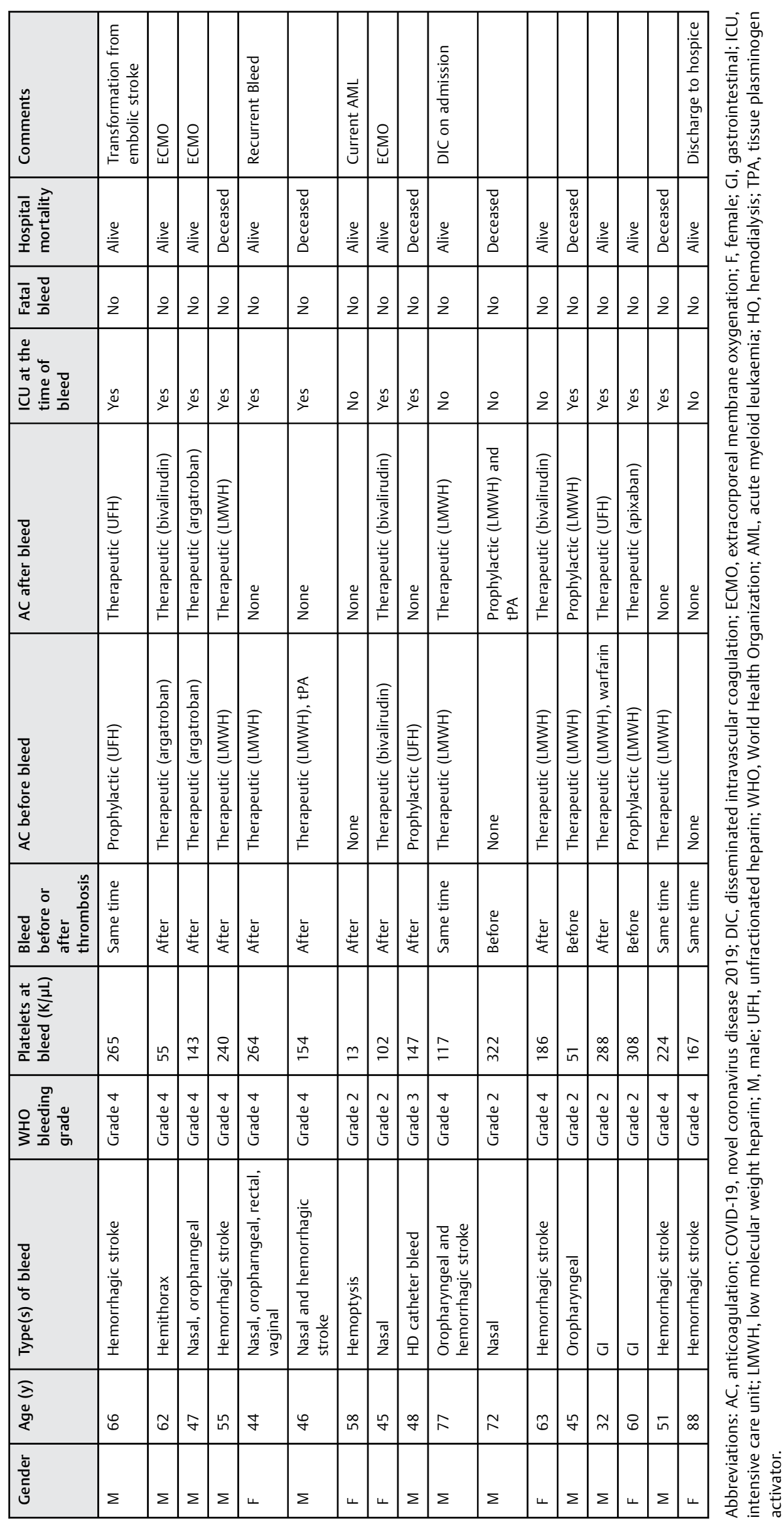


thrombocytopenia defined as platelet count $<100 \times 10^{3} \mu \mathrm{L}$ at the time of bleeding.

Our study showed increased incidence of thrombosis in hospitalized COVID-19-positive patients. These patients also had longer hospital LOS and increased mortality. Risk factors identified for thrombosis include older age and Hispanic ethnicity. Analysis of hospital course showed patients with D-dimer $>6 \mathrm{x}$ ULN, ICU admission, RRT requirement, and mechanical ventilation had higher incidence of thrombosis and higher mortality. A risk stratification system based on these factors to identify COVID-19-positive patients who are at high risk for thrombosis may be beneficial for early identification and intervention to reduce hypercoagulability-associated mortality rate in hospitalized COVID-19 patients.

We also found that the incidence of thrombosis was significantly less in patients who received any kind of steroids which was not the case with other COVID-19 therapies. When adjusting for demographics and severity of disease, this was not statistically significant. This could be because this study looked at patients in the first round of the pandemic where only severely sick ICU patients were receiving COVID-19-related therapies and because there were differences in steroid doses given among patients. The RECOVERY trial demonstrated reduced mortality in COVID19 patients who received dexamethasone, and our data show decreased incidence of thrombosis with steroids and how thromboembolism is associated with increased mortality rates. $^{10}$ This may suggest that steroids may help reduce mortality by diminishing the proinflammatory state that leads to hypercoagulability. Steroids have been previously shown to increase risk of thrombotic events in non-COVID19 patients, but the pathogenesis of thrombosis in COVID-19 is starkly different than in other disease states. Prospective studies need to be done with standard dosing of steroids in all COVID-19 patients to understand if steroids should be used in COVID-19 patients with high risk for thrombosis.

There was no difference in mortality in COVID-19 patients who had prophylactic versus therapeutic dosing of anticoagulation, even when adjusting for demographics and severity of disease. Our study specifically looked at empiric prophylactic and therapeutic anticoagulation given to COVID-19 patients prior to a thrombotic event to assess mortality benefit. Our study supports society guidelines that prophylactic anticoagulation should be given to all hospitalized COVID-19-positive patients in the absence of confirmed or high suspicion for VTE, but prospective studies need to be done to evaluate if there is further benefit for increased doses of thromboprophylaxis in this high-risk population. ${ }^{6}$

The majority of bleeding events were severe (WHO grade 4) and occurred in the ICU, where patients were thought to be at highest risk for thrombosis and were empirically treated with therapeutic anticoagulation. Given that most patients had normal platelet counts, this bleeding was not attributed to disseminated intravascular coagulation (DIC) which has been commonly noted in COVID-19 patients. ${ }^{8}$ Risk factors, such as stress gastritis, contributed to bleeding in critically ill patients, and long-term morbidity of bleeding events should be taken into consideration. We suggest that there should be careful monitoring for signs and symptoms of bleeding in critically ill COVID-19 patients, while they are on therapeutic anticoagulation.

Our findings support the significant impact of coagulopathy in COVID-19 patients which leads to longer hospital LOS, morbidity, and mortality. There is much work that needs to be done in understanding which patients would most benefit from intermediate to therapeutic dose of anticoagulation while taking into consideration high bleeding rates in this population. Our study also shows that steroids may play a role in reducing thromboembolism in these patients which has not previously been studied. As COVID-19 continues to affect people worldwide, an emphasis needs to be placed on better understanding how to prevent COVID-19-related coagulopathy. Results from randomized control trials are eagerly awaited to provide further guidance in this area.

\section{What Is Known about This Topic?}

There is an increased risk of thrombosis in the novel coronavirus disease 2019 (COVID-19) patients.

- COVID-19 leads to a hypercoagulable state due to increased inflammatory response. Anticoagulation is recommended to decrease risk of thrombosis in COVID-19 patients.

- There are conflicting recommendations whether COVID-19 patients should be on prophylactic versus intermediate versus therapeutic dosing of anticoagulation.

- Low molecular weight heparin is the anticoagulant of choice for COVID-19 patients.

\section{What Does This Paper Add?}

What groups of patients are at highest risk for thrombosis and thrombosis-associated mortality?

- Hispanic patients and older age patients are at higher risk for thrombosis and the novel coronavirus disease 2019 (COVID-19) mortality.

- Patients with D-dimer $>6 \mathrm{x}$ ULN, intensive care unit (ICU) admission, renal replacement therapy (RRT) requirement are more likely to have thrombosis.

- These high risk groups should be closely monitored for signs and symptoms of thrombosis.

Is there any COVID-19 related treatment to reduce risk for thrombosis?

- Patients who received steroids had lower incidence of thrombosis

Should COVID-19 patients get prophylactic versus therapeutic anticoagulation?

- There was no difference in mortality in COVID-19 hospitalized patients who got prophylactic versus therapeutic anticoagulation before thrombosis.

- There were high bleeding rates in COVID-19 patients showing that escalation of anticoagulation does not come without risks. 


\section{Authors' Contributions}

S.W. contributed to writing the first draft of the manuscript, study concept and design, literature review, data collection, data analysis, creation of tables and figures, critical revision of the manuscript, and final approval.

E.B. contributed to study concept and design, data extraction, creation of tables, critical revision of the manuscript, and final approval.

J.T. contributed to data extraction, creation of tables, critical revision of the manuscript, and final approval.

S.G. and P.V. contributed to critical revision of the manuscript and final approval.

S.J. contributed to study concept and design, thorough mentorship and guidance through research process, critical revision of the manuscript, and final approval.

Note

This research was presented in oral presentation form at the 62nd annual meeting of the American Society of Hematology on December 7th, 2020, virtual.

Funding

No outside or third-party funding was used for this study.

\section{Conflicts of Interest}

The authors do not report any disclosures or conflicts of interest related to this manuscript.

\section{Acknowledgments}

The authors would like to acknowledge Ekta Kishen for her contribution to data extraction and Todd Beck for his help with statistical analysis of our data. The authors would also like to thank Dr. Debasish Tripathy for reviewing this manuscript.

\section{References}

1 Singhania N, Bansal S, Nimmatoori DP, Ejaz AA, McCullough PA, Singhania G. Current overview on hypercoagulability in COVID19. Am J Cardiovasc Drugs 2020;20(05):393-403

2 Huang C, Wang Y, Li X, et al. Clinical features of patients infected with 2019 novel coronavirus in Wuhan, China. Lancet 2020;395 (10223):497-506

3 Jiménez D, García-Sanchez A, Rali P, et al. Incidence of VTE and bleeding among hospitalized patients with coronavirus disease 2019: a systematic review and meta-analysis. Chest 2021;159(03):1182-1196

4 Klok FA, Kruip MJHA, van der Meer NJM, et al. Incidence of thrombotic complications in critically ill ICU patients with COVID-19. Thromb Res 2020;191:145-147

5 Barnes GD, Burnett A, Allen A, et al. Thromboembolism and anticoagulant therapy during the COVID-19 pandemic: interim clinical guidance from the anticoagulation forum. J Thromb Thrombolysis 2020;50(01):72-81

6 Cuker A, Tseng EK, Nieuwlaat R, et al. American Society of Hematology 2021 guidelines on the use of anticoagulation for thromboprophylaxis in patients with COVID-19. Blood Adv 2021; 5(03):872-888

7 Lauzier F, Arnold DM, Rabbat C, et al. Risk factors and impact of major bleeding in critically ill patients receiving heparin thromboprophylaxis. Intensive Care Med 2013;39(12):2135-2143

8 Al-Samkari H, Karp Leaf RS, Dzik WH, et al. COVID-19 and coagulation: bleeding and thrombotic manifestations of SARSCoV-2 infection. Blood 2020;136(04):489-500

9 Dreijer AR, Diepstraten J, Brouwer R, et al. Risk of bleeding in hospitalized patients on anticoagulant therapy: prevalence and potential risk factors. Eur J Intern Med 2019;62:17-23

10 Horby P, Lim WS, Emberson JR, et al.RECOVERY Collaborative Group. Dexamethasone in hospitalized patients with COVID-19. N Engl J Med 2021;384(08):693-704 